\title{
Chitosan prevents adhesion during rabbit flexor tendon repair via the sirtuin 1 signaling pathway
}

\author{
QIANG CHEN, HUI LU and HU YANG \\ Department of Hand Surgery, The First Affiliated Hospital, College of Medicine, \\ Zhejiang University, Hangzhou, Zhejiang 310003, P.R. China
}

Received September 17, 2014; Accepted June 5, 2015

DOI: $10.3892 / \mathrm{mmr} .2015 .4007$

\begin{abstract}
Chitosan has been demonstrated to exert potent anti-adhesive activity during tendon repair; however, the underlying molecular mechanisms remain unclear. The present study aimed to investigate the preventive effects of chitosan on adhesion in rabbit tendon repair, and to investigate the role of the sirtuin (SIRT)1 signaling pathway in this process. A total of 30 rabbits were divided randomly into three equal groups: Group 1, saline treatment; group 2, chitosan treatment; and group 3 , chitosan + nicotinamide treatment. The flexor tendon of each of the rabbits was injured, and subsequently each rabbit was injected with the one of the reagents. Six weeks post-surgery, all of the rabbits were sacrificed and their flexor tendons were harvested for subsequent evaluation of adhesion. Western blotting was used to determine the protein expression levels of specific signaling molecules. An MTT assay was conducted to evaluate the viability of human tenocytes and flow cytometry was used to analyze the apoptotic rate of the cells. The present study demonstrated that treatment with chitosan relieved adhesion in the rabbits with flexor tendon injuries. In addition, chitosan treatment increased SIRT1 expression, and reduced acetylated p65 and p53 expression in the tendons. The effects of chitosan on the tendons were attenuated by treatment with nicotinamide (a SIRT1 inhibitor). In the human tenocytes, pretreatment with chitosan resulted in an inhibition of interleukin (IL)-1 $\beta$-induced apoptosis. Furthermore, chitosan reversed the IL-1 $\beta$-induced downregulation of SIRT1 and upregulation of acetylated p65 and p53. Furthermore, downregulation of SIRT1 by RNA interference abrogated the effects of chitosan on the levels of p65 and p53 acetylation, and the rate of tenocyte apoptosis. In conclusion, chitosan treatment prevented adhesion via the SIRT1 signaling pathway during rabbit flexor tendon repair. These results indicate that SIRT1 may be targeted for therapeutic intervention in flexor tendon injury.
\end{abstract}

Correspondence to: Dr Qiang Chen, Department of Hand Surgery, The First Affiliated Hospital, College of Medicine, Zhejiang University, 79 Qingchun Road, Hangzhou, Zhejiang 310003, P.R. China

E-mail: qchen8888@163.com

Key words: chitosan, sirtuin 1, flexor tendon, nuclear factor- $-\mathrm{B}, \mathrm{p} 53$

\section{Introduction}

Tendons are dense connective tissues, which are responsible for transferring forces generated by muscles to the opposite side of the joint, and supporting normal movement and stability $(1,2)$. Tendons are commonly subjected to injury, due to various causes (3). A clinical issue associated with tendon repair is the formation of adhesions between a tendon and the surrounding synovial sheath, which may seriously affect the recovery of tendon function. Therefore, tendon adhesion is an important clinical issue (4). The treatment methods used to reduce adhesion in injured flexor tendons include the use of anti-inflammatory agents (5), hyaluronan (6), electric fields (7) and ultrasound (8). The use of biomaterials for the prevention of adhesion has recently been widely studied in the clinical setting. For example, biocompatible phospholipid polymer MPC and hyaluronan have been applied for tendon repair $(9,10)$. However, the underlying cellular and molecular mechanisms of biomaterial treatment of tendon repair remain to be elucidated in order to improve therapeutic methods.

Chitosan, which is derived by partial deacetylation of chitin from crustacean shells, is a polysaccharide copolymer that consists of glucosamine [ $\beta$-(1-4)-linked 2-amino-2-deoxy-D-glucose] and $\mathrm{N}$-acetylglucosamine (2-a cetamido-2-deoxy-D-glucose) $(11,12)$. Due to its biological sensitivity and security, chitosan has been implemented in antimicrobial and antitumor treatment, immune modification, and has been applied in tissue engineering as a bio-scaffold to allow skin or bone cell growth (13). Previous studies have demonstrated the therapeutic action of chitosan on tendon repair, including inhibition of fibroblast growth (14), improved adhesive capacity (15), and cell proliferation and collagen production (16).

Previous studies have demonstrated the regulatory role of chitosan on tendon adhesion; however, the underlying mechanism remains to be fully elucidated. Sirtuin (SIRT) 1 is an $\mathrm{NAD}^{+}$-dependent histone deacetylase for numerous histone and non-histone substrates, which participate in various physiological functions, including cell proliferation, apoptosis and inflammation (17). SIRT1 has previously been reported to be essential for the inhibition of apoptosis and inflammatory responses in human tenocytes (18), thus suggesting that SIRT1 may be an effective therapeutic target for adhesion prevention. However, information regarding the role of SIRT1 signaling 
in tendon repair is considered to be insufficient, particularly in vivo.

In the present study, a rabbit flexor tendon injury model and human tenocytes were used to evaluate the effects of chitosan on tendon adhesion, and investigate the underlying mechanism. In the present study, a rabbit flexor tendon injury model and human tenocytes were used to evaluate the effects of chitosan on tendon adhesion. Whether SIRT1 and its downstream signaling are involved in the anti-adhesion action of chitosan were also investigated.

\section{Materials and methods}

Animals and surgery. A total of 30 mature male New Zealand white rabbits (age, 6 months; weight, $1.84 \pm 0.89 \mathrm{~kg}$ ), purchased from the Animal Breeding Center (Zhejiang University, Hanhzhou, China), were randomly divided into three equal groups: Group 1, saline treatment; group 2, chitosan treatment (Sigma-Aldrich, St. Louis, MO, USA); and group 3, chitosan + nicotinamide treatment (nicotinamide is an inhibitor of SIRT1; Sigma-Aldrich). The left hind limbs of the rabbits in each group were designated as the injured tendon, and the right hind limbs were designated as the normal flexor tendons for each group. The rabbits were maintained in individual standard rabbit cages, with access to a standard rabbit diet and water ad libitum. The study was approved by the ethics committee of The First Affiliated Hospital of Medical School of Zhejiang University (Hangzhou, China).

During surgery, the rabbits were administered an intramuscular injection of $2 \%$ xylazine ( $1 \mathrm{mg} / \mathrm{kg}$; Sigma-Aldrich) as premedication, and 10\% Ketamin-HCl (60 mg/kg; SigmaAldrich) for anesthesia. The thigh of the rabbit was bound using an elastic bandage as a tourniquet, and a $2-\mathrm{cm}$ longitudinal incision was made in the plantar skin between the proximal and distal interphalangeal joints of the toes. The flexor digitorum profundus tendons were transected using a blade, through the incision in the tendon sheath. Finally, the injured tendons were surgically repaired using 6-0 sutures (Hanhzhou AiPu Medical Instrument, Co., Ltd., Hangzhou, China) and the skin covering the lesion was sealed with a simple continuous pattern, using non-absorbable 5-0 silk sutures (Hanhzhou $\mathrm{AiPu}$ Medical Instrument, Co., Ltd). A rectangular window was created in the cast at the site of injury (plantar surface of the hind limb) for injection of the reagents. At $6 \mathrm{~h}$ post-surgery, $0.2 \mathrm{ml}$ saline, $0.2 \mathrm{ml}$ chitosan, or $0.2 \mathrm{ml}$ chitosan $+5 \mathrm{mM} / \mathrm{kg}$ nicotinamide was injected into the hind limb, through the rectangular window, once per day for six weeks.

Adhesion and mechanical assessment. At 6 weeks post-surgery, the rabbits ( $\mathrm{n}=10 /$ group) were sacrificed via $10 \mathrm{ml}$ air via the ear intravenously and the flexor tendons were harvested and stored at $-20^{\circ} \mathrm{C}$ for subsequent morphological and histological analyses. Gross and histological evaluation of tendon adhesion was initially performed. The standard degree of adhesion was determined, as mentioned previously $(12,19)$. Gross evaluation for adhesion degree was determined as follows: None (no adhesions), filmy (separable from the surrounding tissue), mild (not separable from the surrounding tissue), moderate $(35-60 \%$ of the injured area) and severe $(>60 \%$ of the injured area). Evaluation was performed by an investigator in a blinded-manner. Histological evaluation for adhesion formation was determined as follows: None (no adhesions), mild ( $<33 \%$ of the tendon), moderate $(33-66 \%$ of the tendon surface) and severe ( $>66 \%$ of the tendon surface). The average grade from the grades of 10 slides was calculated. In addition, the flexor tendons were fixed on biomechanical instruments, in order to assess mechanical strain. Pretension was set at $10 \mathrm{~N}$ and the maximum tensile breakage of the tendons was recorded at a $20 \mathrm{~mm} / \mathrm{min}$ drawing speed.

Cell lines, culture conditions and treatment. Human tenocytes were derived from five healthy toe tendon explants, which were collected fresh from surgery at the First Affiliated Hospital of Zhenjiang University. In brief, the tendons were stripped aponeurosis under a microscope (DM5500 B; Leica Microsystems, Wetzlar, Germany) and were washed with Hank's solution. The tendon was then subjected to digestion with $0.25 \%$ trypsin and $0.1 \%$ collagenase for $20 \mathrm{~min}$ at of $37^{\circ} \mathrm{C}$. The digested tendons were collected, washed with Hank's solution and cut into $1-2 \mathrm{~mm}^{3}$ fragments. The fragments were exposed to $0.25 \%$ trypsin and $0.1 \%$ collagenase for $1 \mathrm{~h}$ at $37^{\circ} \mathrm{C}$ in order for second digestion. The digested mixture was filtered and centrifuged. The sediment was washed and made into a cell suspension. The cells were counted and inoculated at $5 \times 10^{5} / \mathrm{ml}$. Finally, the cells were cultured in phenol red-free Dulbecco's modified Eagle's medium (DMEM) supplemented with $10 \%$ charcoal stripped fetal bovine serum prior to the subsequent experimental protocols The cells were pretreated with chitosan $(5,10$ or $50 \mu \mathrm{g} / \mathrm{ml})$ for $30 \mathrm{~min}$, and were then split into two groups, those that were activated by interleukin (IL) $-1 \beta(10 \mathrm{ng} / \mathrm{ml})$, and those that were not. High purity chitosan and IL-I $\beta$ were purchased from Sigma-Aldrich, dissolved in normal saline and added to the culture medium according to the indicated concentrations. All reagents were obtained from Invitrogen Life Technologies, (Carlsbad, CA, USA). All subsequent experiments were conducted $1 \mathrm{~h}$ after IL-1 $\beta$ supplementation, unless otherwise stated.

MTT viability assay. The effects of chitosan and IL-1 $\beta$ on cell viability were detected using an MTT assay (Sigma-Aldrich), which is based on the uptake of 3-(4,5-dimethylthiazol-2-yl)2,5-diphenyltetrazolium bromide. The tenocytes ( $2 \times 10^{3}$ cells/well) were cultured in 96 -well plates $24 \mathrm{~h}$ prior to treatment with chitosan. Following treatment with the reagents, the MTT solution was added to each well and incubated for $2 \mathrm{~h}$ at $37^{\circ} \mathrm{C}$. SDS buffer (10\%) supplemented with $0.01 \mathrm{M} \mathrm{HCl}$ was then added to the cells and left for $12 \mathrm{~h}$. Subsequently, the absorbance at $570 \mathrm{~nm}$ was measured using a spectrophotometer (Perkin Elmer LS-55; PerkinElmer Inc., Waltham, MA, USA). Independent experiments were performed in triplicate.

Western blot analysis. The rabbit flexor tendons or human tenocytes were lysed using ice-cold lysis buffer containing: $50 \mathrm{mmol} / 1$ Tris- $\mathrm{HCl}$ (pH 7.4); 1\% NP-40; $150 \mathrm{mmol} / \mathrm{l} \mathrm{NaCl} ; 1 \mathrm{mmol} / \mathrm{l}$ EDTA; 1 mmol/l phenylmethylsulfonyl fluoride; and complete proteinase inhibitor mixture (one tablet/10 ml; Roche Molecular Biochemicals, Indianapolis, IN, USA). A Bicinchoninic Acid Protein Assay kit (Beyotime Institute of Biotechnology, Haimen, China) was used to quantify the protein concentration within the lysate and western blot analysis was subsequently performed. 
Table I. Gross and histological evaluation of tendon adhesion.

\begin{tabular}{|c|c|c|c|c|c|c|c|c|c|}
\hline \multirow[b]{2}{*}{ Group } & \multicolumn{5}{|c|}{ Gross evaluation of tendon adhesion } & \multicolumn{4}{|c|}{ Histological degree of tendon adhesion } \\
\hline & Severe & Moderate & Mild & Filmy & None & Severe & Moderate & Mild & None \\
\hline 1 & 6 & 3 & 1 & - & - & 6 & 3 & 1 & - \\
\hline 2 & - & 1 & 3 & 6 & - & - & 2 & 7 & 1 \\
\hline 3 & 5 & 4 & 1 & - & - & 5 & 3 & 2 & - \\
\hline
\end{tabular}

Group 1, injected with normal saline; group 2, injected with chitosan; group 3, injected with chitosan + nicotinamide. Filmy, tendons separable from surrounding tissue.

Proteins $(40 \mu \mathrm{g})$ were separated by $10 \%$ SDS-PAGE and transferred to a nitrocellulose membrane (EMD Millipore, Bilerica, MA, USA). The membrane was then probed with mouse antigoat polyclonal antibody against SIRT1 (cat. no. sc-19857; Santa Cruz Biotechnology, Inc., Dallas, TX, CA), rabbit polyclonal antibody against acetylated p65 (cat. no. 3045s; Cell Signaling Technology, Inc., Beverly, MA, USA), rabbit polyclonal antibody against acetylated p53 (cat. no. 2570s; Cell Signaling Technology, Inc) and rabbit polyclonal antibody against $\beta$-actin (cat. no. A2066; Sigma-Aldrich), followed by incubation with anti-goat (cat. no. ab157532 ) or anti-rabbit (cat. no. ab191866) secondary antibodies (Abcam, Cambridge, MA, USA). All antibodies were used at concentrations and dilutions as recommended by the manufacturer's instructions (dilutions ranged between 1:100 and 1:10,000 for western blot analysis). The blots were visualized by enhanced chemiluminescence (ECL) using ECL reagent (Pierce Biotechnology, Inc., Rockford, IL, USA) and images were captured on X-ray films (ChampGel 6000; Beijing Sage Creation Science, Co., Ltd., Beijing, China).

Apoptosis analysis. Detection and quantification of apoptosis was performed using flow cytometry. After treatment with the reagents, the tenocytes were cultured with binding buffer, Annexin V-Enhanced Green Fluorescent Protein and propidium iodide (Sigma-Aldrich). The mixture was then incubated at room temperature for $15 \mathrm{~min}$ in the dark, followed by flow cytometry (FACScan; BD Biosciences, Franklin Lakes, NJ, USA). The percentage of apoptotic cells was quantified using CellQuest software version 5.1 (BD Biosciences).

Inhibition of SIRT1 by RNA interference. Double-stranded oligonucleotides 5'-GATCCCGTTGGATGATATGACACT GTTCAAGAGACAGTGTCATATCATCCAACTTTTTTG GAAA-3' (SIRT1 target sequence underlined) were cloned into the pSuperiorRetroPuro vector (Oligoengine, Seattle, WA, USA). The plasmid was packaged into a retrovirus by transfection of the amphotropic packaging cell line LA (Cell Biolabs, Inc. USA). A virus expressing a scrambled small interfering (si)RNA (5'-GATCCCGCCGTCGTCGATAAGCAATATTTGATATC CGATATTGCTTATCGACGACGGCTTTTTTA-3'; pSuperiorRetroPuro vector) served as a control. Human tenocytes were infected with the SIRT1 siRNA retrovirus and selected using $0.5 \mathrm{~g} / \mathrm{ml}$ puromycin (Sigma-Aldrich) for 10 days. Cells were harvested for the subsequent apoptotic assay and western blot analysis.

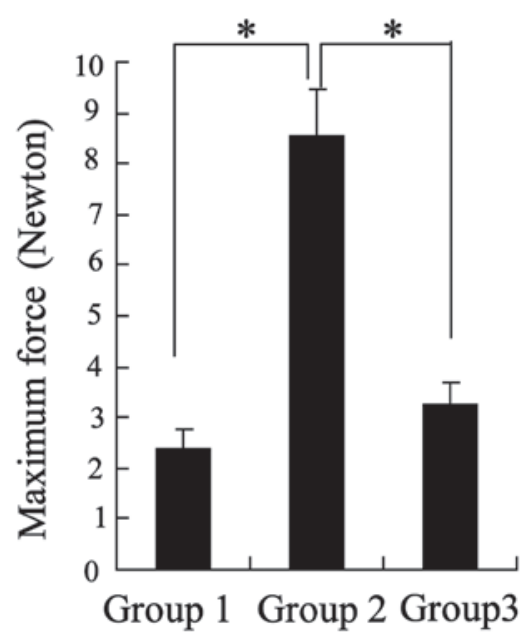

Figure 1. Effects of chitosan on the maximum force of tendon anastomosis in the injured flexor tendons of rabbits. Rabbit flexor tendons were injured and subsequently injected with normal saline (group 1), chitosan (group 2) or chitostan + nicotinamide (group 3 ). Data are presented as the mean \pm standard deviation. ${ }^{*} \mathrm{P}<0.05$

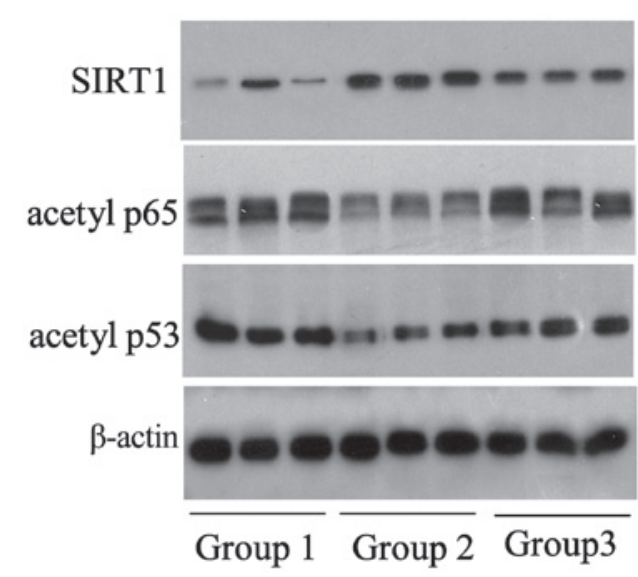

Figure 2. Treatment with chitosan upregulated SIRT1, and downregulated acetylation of p65 and p53 in rabbit flexor tendons. Following injury, the tendons were injected with saline (group 1), chitosan (group 2) or chitosan + nicotinamide (group 3). The expression of SIRT1, acetylated p65 and acetylated p53 in the rabbit flexor tendons was analyzed by western blotting. Each group underwent three tests. SIRT1, sirtuin; acetyl, acetylated.

Statistical analysis. Statistical analysis was performed using SPSS 16.0 statistical analysis software (SPSS Inc., Chicago, 
A

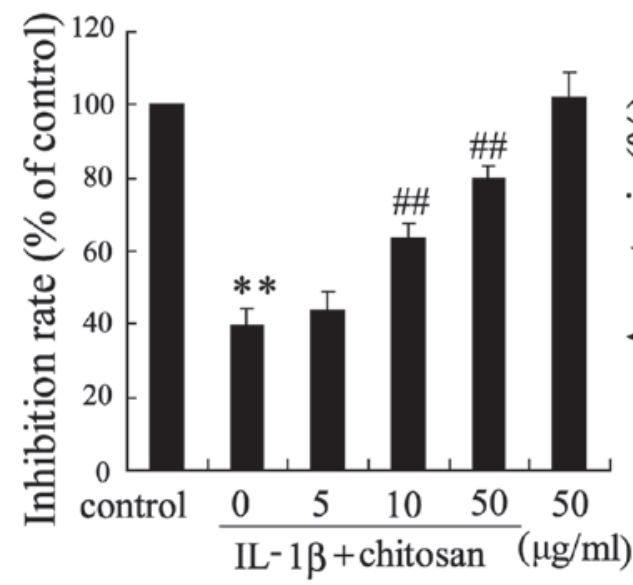

B

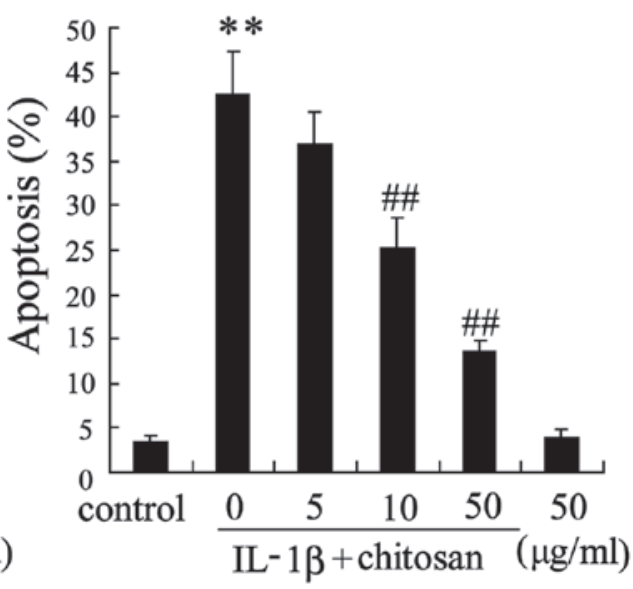

Figure 3. Effects of chitosan on IL-1 $\beta$-induced (A) cell viability and (B) apoptosis in human tenocytes. The cells were pretreated with chitosan ( $0,5,10$ or $50 \mu \mathrm{g} / \mathrm{ml})$ and stimulated by IL-1 $\beta(10 \mathrm{ng} / \mathrm{ml})$. Data are presented as the mean \pm standard deviation. ${ }^{* *} \mathrm{P}<0.05$ compared with the control group, ${ }^{\# \#} \mathrm{P}<0.05$ compared with the IL-1 $\beta$-treated group. IL-1 $\beta$, interleukin-1 $\beta$.

IL, USA). Data was analyzed using one-way analysis of variance. Statistical analyses of cell viability and apoptosis were conducted using Student's t-test. All data were expressed as the mean \pm standard deviation. $\mathrm{P}<0.05$ was considered to indicate a statistically significant difference.

\section{Results}

Adhesion formation. Following surgery, the flexor tendons were harvested from the rabbits, in order to determine the state of adhesion. Based on the gross (10) and histological evaluations of adhesion (Table I), the numbers of rabbits exhibiting severe and moderate levels of adhesion werelowest in the chitosan-treated rabbits (group 2; 0 severe and 2 moderate; $\mathrm{n}=10$ ), when compared with the levels in the normal saline-treated rabbits (group 1; 6 severe and 3 moderate; $\mathrm{n}=10$ ), and the effect of chitosan was reversed following nicotinamide treatment (group 3; 5 severe and 3 moderate; $n=10$ ). Furthermore, analysis of the maximum force generated by the tendon anastomoses demonstrated that the greatest forces were observed in the chitosan group (Fig. 1). These results indicated that chitosan may possess anti-adhesive bioactivity.

Expression levels of signaling molecules. In addition to examination of the physiological adhesion status, the flexor tendons were randomly selected and disrupted using liquid nitrogen, in order to detect the expression levels of specific signaling molecules. As shown in Fig. 2, the expression levels of SIRT1 in the chitosan group (group $2 ; n=3 /$ group) were elevated, when compared with the control group; chitosan-induced SIRT1 protein expression was observed to be inhibited by nicotinamide. Conversely, the expression levels of the SIRT1-targeted proteins, acetylated p65 and p53, decreased with the upregulation of SIRT1 and increased with the downregulation of SIRT1, which was induced by nicotinamide.

Chitosan reverses $I L-1 \beta$-induced proliferation and apoptosis of human tenocytes. An in vitro experiment was performed in human tenocytes to further ascertain the protective effects of

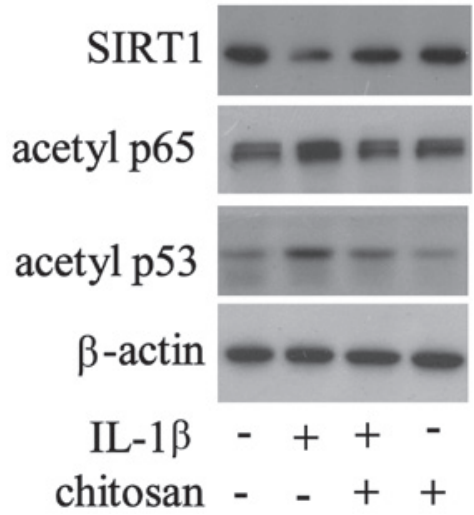

Figure 4. Effects of chitosan on IL-1 $\beta$-induced upregulation of SIRT1, and downregulation of acetyl p65 and p53 in human tenocytes. The cells were pretreated with chitosan $(50 \mu \mathrm{g} / \mathrm{ml})$ and stimulated with IL-1 $\beta$ (10 ng/ml). Protein expression was detected by western blotting. SIRT1, sirtuin; acetyl, acetylated; IL-1 $\beta$, interleukin-1 $\beta$.

chitosan on tendon repair. Following chitosan pretreatment, the tenocytes were incubated with IL-1 $\beta$. Fig. 3 demonstrates that chitosan influenced the survival of tenocytes. Treatment with $10 \mu \mathrm{g} / \mathrm{ml}$ or $50 \mu \mathrm{g} / \mathrm{ml}$ chitosan attenuated IL-1 $\beta$-induced cell proliferation (Fig. 3A) and apoptosis (Fig. 3B) in a dose-dependent manner, compared with the IL-1 $\beta$ group.

Chitosan attenuates IL-1 $\beta$-induced expression of signaling proteins in human tenocytes. Following chitosan pretreatment, the cells were exposed to IL-1 $\beta$, and the expression levels of various signaling molecules were detected. As shown in Fig. 4, the expression levels of SIRT1 were downregulated and the expression levels of acetylated p65 and p53 were upregulated in the tenocytes stimulated with IL-1 $\beta$. However, chitosan reversed the changes in protein expression that had been induced by IL-1 $\beta$. These results demonstrate the fundamental regulatory role of SIRT1 in tendon repair by chitosan.

Knockdown of SIRT1 reduces the bioactivity of chitosan on human tenocytes. To determine the role of SIRT1 in the 

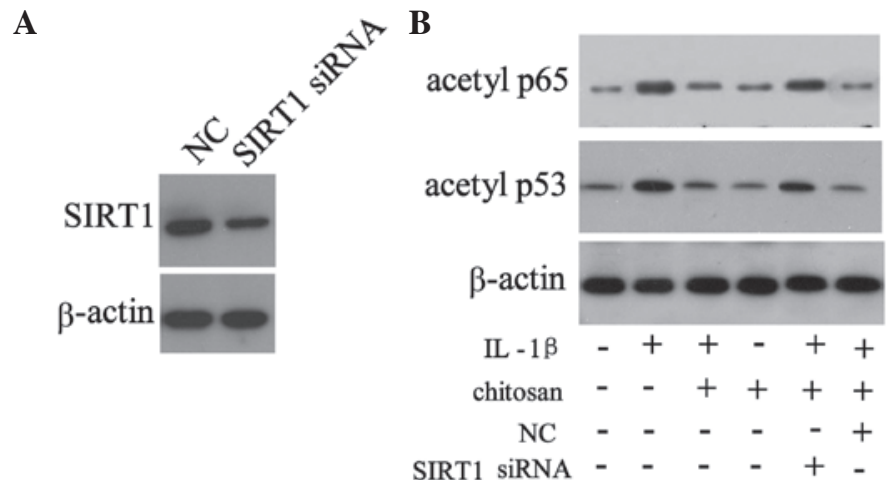

C

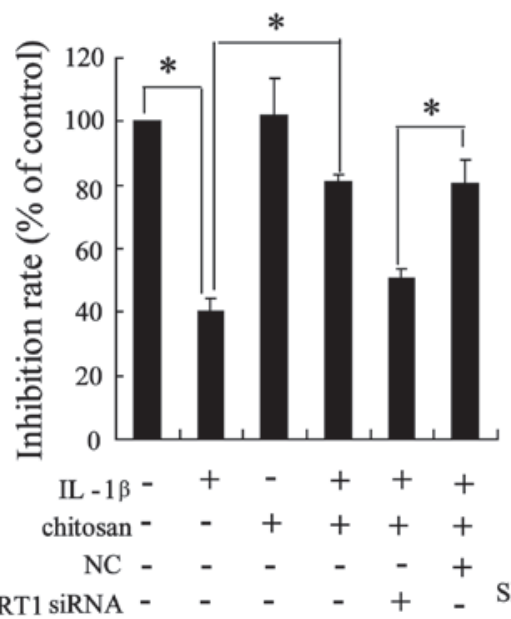

D

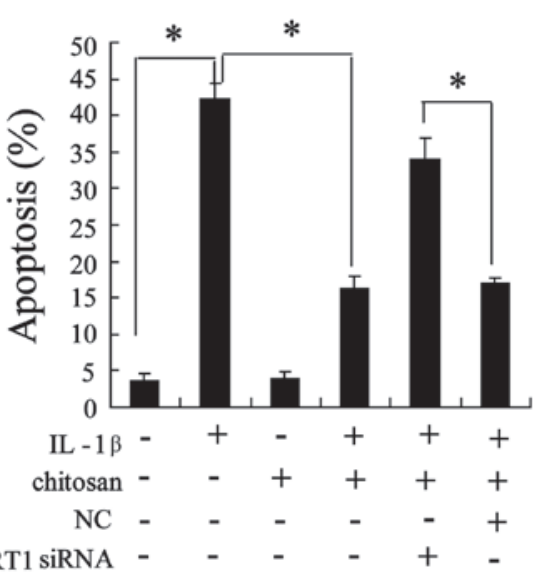

Figure 5. Knockdown of SIRT1 reversed the bioactivity of chitosan on human tenocytes. (A) The cells were transfected with si-SIRT1 and the expression of SIRT1 was detected. Transfected cells were treated with chitosan $(50 \mu \mathrm{g} / \mathrm{ml})$ and IL-1 $\beta(10 \mathrm{ng} / \mathrm{ml})$ to detect the effects on (B) p65 and p53 acetylation, (C) cell viability and (D) apoptosis. Data are presented as the mean \pm standard deviation. " $\mathrm{P}<0.05$ compared with the control groups. SIRT1, sirtuin; si, small interfering; IL-1 $\beta$, interleukin-1 $\beta$; acetly, acetylated; NC, negative control.

protection of tendons from adhesion, the tenocytes were transfected with small interfering (si)-SIRT1. As shown in Fig. 5A, transfection with si-SIRT1 resulted in a downregulation of SIRT1 expression, indicating the successful knockdown of SIRT1. As shown in Fig. 4, chitosan reversed the IL-1 $\beta$-induced downregulation of acetylated p65 and p53. Notably, the action of chitosan on the IL-1 $\beta$-induced expression of acetylated p65 and p53 was abrogated following SIRT1 knockdown (Fig. 5B). Furthermore, silencing of SIRT1 expression abolished the effects of chitosan on IL-1 $\beta$-induced cell proliferation (Fig. 5C) and apoptosis (Fig. 5D), when compared with the IL-1 $\beta+$ chitosan negative control group.

\section{Discussion}

Adhesion formation is a common clinical problem, which is characteristic of alignment and maturation of tenoblasts and collagen fibers, and inadequate attachment of the tendon to its location (20). It has previously been demonstrated that the prevention of tendon adhesion is an important objective of hand surgery (21). The results of the present study demonstrate that administration of chitosan relieves the structural and biomechanical properties during an experimental model of flexor tendon wound repair. In addition, SIRT1 was observed to be a key regulator affected by chitosan treatment in the prevention of tendon adhesion.
Tendon repair may occur intrinsically, via proliferation of epitenon and endotenon tenocytes, which results in improved biomechanics and fewer complications; or extrinsically, via invasion of cells from the surrounding sheath and synovium, which causes adhesion formation (22). It has previously been reported that inflammation is a common initial event of tendon repair. During inflammation of the tendons, vasoactive and chemotactic factors are released resulting in increased vascular permeability, initiation of angiogenesis, stimulation of tenocyte proliferation, and recruitment of more inflammatory cells (23). It has been suggested that protective tenocytes, released during inflammation in intrinsic repair, may be effective in the prevention of tendon adhesion. Accordingly, the present study demonstrated that chitosan exerted an anti-adhesive effect on flexor tendons, and abolished tenocyte apoptosis, which had been induced by IL-1 $\beta$ (an inflammatory factor). Chitosan is a type of biomaterial that possesses hemostatic and anti-inflammatory properties, which has been shown (experimentally and clinically) to prevent adhesion (24). However, the underlying mechanism of the anti-adhesive effect of chitosan and the associated intracellular signaling pathway has yet to be fully elucidated.

SIRT1 has been identified as a modulator in the development and progression of inflammation through the deacetylation of histones and critical transcription factors, thus leading to transcriptional repression of various inflammation-associated genes (17). In addition, SIRT1 has been shown to inhibit apoptosis and the inflammatory response of tenocytes (18). 
Therefore, the present study examined whether SIRT1 signaling may be involved in the prevention of adhesion by chitosan treatment in rabbit flexor tendons and human tenocytes. The results indicated that chitosan increased the expression of SIRT1 in adhesive tendons in vivo and reversed the IL-1 $\beta$-mediated downregulation of SIRT1 in tenocytes. In addition, it was demonstrated that inhibition of SIRT1 by nicotinamide partly suppressed the ability of chitosan to prevent adhesion in rabbits. These results indicated a regulatory role of SIRT1 signaling in the anti-adhesive properties of chitosan.

Nuclear factor $(\mathrm{NF})-\kappa \mathrm{B}$ is a nuclear transcription factor, which has been reported to regulate the gene expression of numerous proinflammatory proteins (17). The present study demonstrated that chitosan reversed proinflammatory IL-1 $\beta$-induced upregulation of acetylated p65 (a subunit of NF- $\mathrm{NB}$ ) and upregulated SIRT1. It has previously been reported that deacetylation of $\mathrm{NF}-\kappa \mathrm{B}$ subunit p65 may lead to a decrease in $\mathrm{NF}-\kappa \mathrm{B}$ transcriptional activity, thereby resulting in cell apoptosis $(17,25)$. In this context the results of the present study indicated that knockdown of SIRT1 abrogated the inhibitory effects of chitosan on IL-1 $\beta$-induced p65 acetylation and apoptosis. These results indicate that chitosan may inhibit inflammation-associated NF- $\kappa \mathrm{B}$ activation via SIRT1 signaling during adhesion repair.

The present study also demonstrated that the expression of p53 was upregulated in adhesive tendons and tenocytes that had been exposed to IL-1 $\beta$. As a tumor suppressor gene, p53 is stimulated by various stress signals and is significant in modulating the cell cycle and apoptosis (26,27). p53 has been characterized as one of the numerous substrates of SIRT1, and deacetylation at the lysine residue of $\mathrm{p} 53$ reduces its DNA binding activity, thus affecting co-activator recruitment, which is required for cell survival (28). The present study also demonstrated that chitosan inhibited p53 activation in rabbit adhesive tendons and IL-1 $\beta$-induced tenocytes. In addition, knockdown of SIRT1 resulted in increased acetylation of $\mathrm{p} 53$, as well as increased cell viability and reduced apoptosis in human tenocytes. To the best of our knowledge, the present study is the first to demonstrate a regulatory role of SIRT1 signaling (via NF- $\kappa \mathrm{B}$, a subunit of p53, and $\mathrm{p53}$ ) in the prevention of adhesion using chitosan, in vivo.

In conclusion, the present study provided important insights regarding the underlying mechanisms of adhesion prevention via the administration of chitosan. Prevention of adhesion was shown to be associated with SIRT1 signaling in a flexor tendon repair model. Furthermore, the results indicated that chitosan may inhibit inflammation and protect tenocytes from apoptosis, via suppression of NF- $\mathrm{NB}$ and activation of $\mathrm{p} 53$ by SIRT1. The upregulation of SIRT1 as a result of chitosan treatment in injured tendons may be useful in the development of future therapeutic strategies for the treatment of tendon injury.

\section{References}

1. Moshiri A and Oryan A: Structural and functional modulation of early healing of full-thickness superficial digital flexor tendon rupture in rabbits by repeated subcutaneous administration of exogenous human recombinant basic fibroblast growth factor. J Foot Ankle Surg 50: 654-662, 2011.

2. Chalmers J: Review article: Treatment of Achilles tendon ruptures. J Orthop Surg (Hong Kong) 8: 97-99, 2000.

3. Peterson RK, Shelton WR and Bomboy AL: Allograft versus autograft patellar tendon anterior cruciate ligament reconstruction: A 5-year follow-up. Arthroscopy 17: 9-13, 2001.
4. Woo SL, Lee TQ and Abramowitch SD: Structure and function of ligaments and tendons. In: Basic Orthopaedic Biomechanics and Mechano-biology. Mow VC and Huiskes R (eds.). 3rd edition. Lippincott Williams \& Wilkins, Philadelphia, pp301-342, 2005.

5. Kulick MI, Smith S and Hadler K: Oral ibuprofen: Evaluation of its effect on peritendinous adhesions and the breaking strength of a tenorrhaphy. J Hand Surg Am 11: 110-120, 1986.

6. Amiel D, Ishizue K, Billings E Jr, Wiig M, Vande Berg J, Akeson WH and Gelberman R: Hyaluronan in flexor tendon repair. J Hand Surg Am 14: 837-843, 1989.

7. Fujita M, Hukuda S and Dodia Y: The effect of constant direct electrical current on intrinsic healing in the flexor tendon in vitro. An ultrastructural study of differing attitudes in epitenon cells and tenocytes. J Hand Surg Br 17: 94-98, 1992.

8. Turner SM, Powell ES and Ng CS: The effect of ultrasound on the healing of repaired cockerel tendon: Is collagen cross-linkage a factor? J Hand Surg Br 14: 428-433, 1989.

9. Ishiyama N, Moro T, Ohe T, Miura T, Ishihara K, Konno T, Ohyama T, Kimura M, Kyomoto M, Saito T, Nakamura K and Kawaguchi H; Reduction of Peritendinous adhesions by hydrogel containing biocompatible phospholipid polymer MPC for tendon repair. J Bone Joint Surg Am 93: 142-149, 2011.

10. Liu Y, Skardal A, Shu XZ and Prestwich GD. Prevention of peritendinous adhesions using a hyaluronan-derived hydrogel film following partial-thickness flexor tendon injury. J Orthop Res 26: 562-569, 2008

11. Illum L: Chitosan and its use as a pharmaceutical excipient. Pharm Res 15: 1326-1331, 1998.

12. Raafat D and Sahl HG: Chitosan and its antimicrobial potential - a critical literature survey. Microb Biotechnol 2: 186-201, 2009.

13. Kaats GR, Michalek JE and Preuss HG: Evaluating efficacy of a chitosan product using a double-blinded, placebo-controlled protocol. J Am Coll Nutr 25: 389-394, 2006.

14. Zhang H, Sheng ZJ and Hou CL: Effect of chitosan membrane on tendon adhesion and healing. Zhongguo Xiu Fu Chong Jian Wai Ke Za Zhi 13: 382-385, 1999 (In Chinese).

15. Majima T, Funakosi T, Iwasaki N, Yamane ST, Harada K, Nonaka S, Minami A and Nishimura S: Alginate and chitosan polyion complex hybrid fibers for scaffolds in ligament and tendon tissue engineering. J Orthop Sci 10: 302-307, 2005.

16. Xia CS, Hong GX, Dou RR and Yang XY: Effects of chitosan on cell proliferation and collagen production of tendon sheath fibroblasts, epitenon tenocytes, and endotenon tenocytes. Chin J Traumatol 8: 369-374, 2005.

17. Yeung F, Hoberg JE, Ramsey CS, Keller MD, Jones DR, Frye RA and Mayo MW: Modulation of NF-kappaB-dependent transcription and cell survival by the SIRT1 deacetylase. EMBO J 23: 2369-2380, 2004.

18. Busch F, Mobasheri A, Shayan P, Stahlmann R and Shakibaei M: Sirt-1 is required for the inhibition of apoptosis and inflammatory responses in human tenocytes. J Biol Chem 287: 25770-25781, 2012.

19. Tang JB, Shi D and Zhang QG: Biomechanical and histologic evaluation of tendon sheath management. J Hand Surg Am 21: 900-908, 1996.

20. Sharma $P$ and Maffulli N: Tendon injury and tendinopathy: Healing and repair. J Bone Joint Surg Am 87: 187-202, 2005.

21. Khanna A, Gougoulias N and Maffulli N: Modalities in prevention of flexor tendon adhesion in the hand: what have we achieved so far? Acta Orthop Belg 75: 433-444, 2009

22. Gelberman RH, Manske PR, Vande Berg JS, Lesker PA and Akeson WH: Flexor tendon repair in vitro: A comparative histologic study of the rabbit, chicken, dog, and monkey. J Orthop Res 2: 39-48, 1984.

23. Sharma Pand MaffulliN: Biology of tendon injury: Healing, modeling and remodeling. J Musculoskelet Neuronal Interact 6: 181-190, 2006.

24. Moutzouri AG and Athanassiou GM: Insights into the alteration of osteoblast mechanical properties upon adhesion on chitosan. Biomed Res Int 2014: 740726, 2014.

25. Tak PP, Gerlag DM, Aupperle KR, van de Geest DA, Overbeek M, Bennett BL, Boyle DL, Manning AM and Firestein GS: Inhibitor of nuclear factor kappaB kinase beta is a key regulator of synovial inflammation. Arthritis Rheum 44: 1897-1907, 2001.

26. Ryan KM, Phillips AC and Vousden KH: Regulation and function of the p53 tumor suppressor protein. Curr Opin Cell Biol 13: 332-337, 2001

27. Vogelstein B, Lane D and Levine AJ: Surfing the p53 network. Nature 408: 307-310, 2000.

28. Sakaguchi K, Herrera JE, Saito S, Miki T, Bustin M, Vassilev A, Anderson CW and Appella E: DNA damage activates p53 through a phosphorylation-acetylation cascade. Genes Dev 12: 2831-2841, 1998 